\title{
Aproximación del arte de acción a la Educación Infantil. Otras formas de interrelación en el contexto educativo
}

\section{Approaches of the Action Art in early childhood Education. Some other ways of interrelation within the educational system}

\author{
B. Soledad SÁnchez-Arenas \\ Universidad de Murcia \\ bibianasoledad.sanchez@um.es \\ P. Manuela Soto-Solier \\ Universidad de Murcia \\ pm.sotosolier@um.es
}

Recibido: 5 de septiembre de 2013

Aprobado: 17 de marzo de 2016

\begin{abstract}
Resumen
Este trabajo de investigación artística, se crea con el propósito de experimentar sobre cómo llevar a la práctica aproximaciones entre el arte contemporáneo y la vida cotidiana universitaria. En este caso, más concretamente, entre el arte de acción y el alumnado de Educación Infantil de la Facultad de Educación de Alicante. El desconocimiento generalizado sobre los cambios acontecidos en el arte durante el siglo $\mathrm{XX}$, evidencia la falta de interés del alumnado sobre el arte contemporáneo, y por consiguiente, sigue estando vigente, la separación entre arte y vida. Por ello, como artistas y docentes, abrimos la posibilidad de realizar experimentos concretos dentro de la vida cotidiana educativa, para que a través del arte de acción, se llegue a una interacción comunicativa como proceso activo de aprendizaje, y así, cambiar las relaciones habituales que se tienen en un contexto determinado, con el otro y consigo mismo, generando futuros consumidores y transmisores de arte contemporáneo.
\end{abstract}

Palabras clave: arte de acción, educación artística, arte=vida, interrelación comunicativa.

Sánchez-Arenas, B.S., Soto-Solier, P.M. (2016): Aproximación del arte de acción a la Educación Infantil. Otras formas de interrelación en el contexto educativo. Arte, Individuo y Sociedad, 28(2) 183-200

\begin{abstract}
This Artistic research project was created in order to test how to put into practice approaches between Contemporary Art and University daily life. In this particular case, between Action Art and the students at the Early Childhood Education University in Alicante. The generalized lack of awareness about changes which took place in Art in the XX century, demonstrates the lack of interest on the part of students about Contemporary Art, and therefore, it is still remarkable, the distance between Art and life. Thus, as artists and teachers, the chance to carry out specific experiments is open within everyday educational life. Therefore, through Action Art a communicative interaction is possible to be achieved as an active learning process and, in such way, change the usual existing relationships in a predetermine context, creating this way, future Contemporary Art consumers and transmitters. Keywords: action art, artistic education, art=life, communicative interrelationship.
\end{abstract}


Sumario: 1. Introducción, 2. Intención artística de unir el arte y la vida cotidiana durante el siglo XX, 3. El arte como expresión social y su necesaria interpretación, 4. Desarrollo de un caso práctico para llevar a cabo posibles aproximaciones entre el arte de acción y la Educación Infantil en la vida cotidiana educativa, 4.1. Contextualización, características del alumno-a/espectador-a y aparatos utilizados para registrar las experiencias, 4.2. Primera fase del proceso, 4.2.1. Performance I, 4.2.2 Pre-test realizado en el contexto cotidiano, 4.2.3 Performance II, 4.2.4 Performance III, Conclusiones de la primera fase del proceso, 4.3 Segunda fase del proceso, 4.3.1 Proceso de desarrollo performativo para los/as alumnos-as/espectadores. Diferencia entre presentación (acción) y representación, 4.3.2 Práctica sobre el concepto presentación, 4.3.3 Práctica sobre el concepto representación, Conclusiones de la segunda fase del proceso, 5. Conclusiones generales. Referencias.

\section{Introducción}

Creemos en la necesidad de seguir generando más espacios de diálogos y participación, entre el arte contemporáneo y el público en general, para facilitar una interacción que permita una mayor comunicación entre ambas partes. Para ello, esta investigación artística empírica, se ha desarrollado en el entorno académico universitario, un contexto social condicionado por las relaciones humanas estereotipadas. La observación y reflexión sobre este ámbito social y educativo, es lo que nos ha motivado a plantear este trabajo de investigación, creando experiencias estéticas interhumanas, sin estar condicionados a un resultado mercantil.

Debido a la complejidad y pluralidad, que se ha ido planteando en el desarrollo de la producción artística contemporánea, nos hemos alejado de realizar análisis exhaustivos y definiciones cerradas y universales, que nos alejarían de nuestra intención, por ello, hemos concretado nuestra investigación, en el arte de acción, para llevar a cabo su aproximación a la vida cotidiana educativa pues consideramos que es una de las tendencias artísticas menos conocidas y que tiene más dificultades para que se pueda llegar a producir una interacción comunicativa, y por consiguiente, una aproximación real entre el arte y la vida y que no se quede en un mero contacto.

Para poder evidenciar el carácter procesual de esta investigación, la hemos divido en tres apartados y, a su vez, el último apartado donde se desarrolla el caso práctico, está subdividido en dos fases:

1. Resumen sobre la intención artística de unir el arte y la vida cotidiana durante el siglo XX.

2. El arte como expresión social y su necesaria interpretación.

3. Se expone la puesta en práctica de la parte empírica de esta investigación, describiendo el trabajo que se ha realizado en el contexto académico y las conclusiones.

\section{Objetivos}

- Realizar aproximaciones entre el arte y la vida cotidiana educativa, para generar más espacios de diálogos y participación entre ambas partes, facilitando así, una interacción que permita una mayor comunicación y que no se quede en un mero contacto.

- Ampliación de los datos cognitivos sobre el arte contemporáneo y más concretamente, sobre el arte de acción. 
- Que el/la alumno-a/espectador-a llegue a entender y asimilar la diferencia entre presentación (acción) y representación, a través, de la vivencia del arte como experiencia de vida.

- Abrir la posibilidad de realizar experiencias artísticas concretas dentro de la vida cotidiana y a través del arte, cambiar las relaciones habituales que se tienen con un contexto determinado, con el otro y consigo mismo.

Metodológicamente, este trabajo de investigación, se ha abordado de forma mixta. Mediante la combinación de la Investigación cualitativa y la cuasiexperimental. García Gallego basándose en los estudios realizados por Campbell y Stanley en 1966, nos indica que unas de las características principales de los diseños cuasiexperimentales (García, 2001), es que se trabaja con grupos formados naturalmente, como es el caso de los grupos con los que trabajamos para esta investigación. También queremos concretar, que aunque desde el punto de vista de la experimentación clásica, los sujetos que participan en la investigación se denominan comúnmente, sujetos experimentales, si bien, para ubicar nomenclaturamente a los participantes en esta investigación, desde el punto de vista artístico en este trabajo, se les denominará formalmente alumnado/espectador.

\section{Intención artística de unir el arte y la vida cotidiana durante el siglo XX}

La noción de "genio" dirigida hacia el artista y desarrollada durante el Renacimiento, provocó el inicio de su separación con los oficios artesanales, a partir de este momento histórico, el artista fue cambiado su estatus en la sociedad. También, sus procesos internos en relación con las transformaciones sociales que comenzaron a finales de siglo XVIII, la ruptura intencionada por parte de éste con el arte decimonónico y su trabajo de investigación dentro de una libertad casi recién estrenada y fomentada, con afirmaciones como las de Kant (1790), "el genio es el talento de producir aquello para lo cual no puede darse ninguna regla" (Efland, 2002, p. 84), fueron propiciando la concepción, que aún persiste en la actualidad, del artista desvinculado de la vida común, una concepción individualista del artista como bohemio, extravagante, genial, incomprendido, atormentado y encerrado en su mundo interior y en su estudio, trabajando sobre cuestiones incompresibles para la mayoría. Aunque este patrón, ya ha quedado caduco y desapareció hace tiempo dentro del ámbito artístico, aún sigue vigente en la percepción del público en general. Esta percepción, sigue abonando la concepción generalizada sobre la subjetividad romántica del artista, creando una diáspora artística separada de la vida.

Partiendo desde esa conciencia de separación entre el arte y la vida cotidiana, para este trabajo de investigación, hemos querido realizar una breve revisión, sobre la intención de algunos artistas del siglo XX para llevar a cabo la fórmula arte $=$ vida, considerándolo desde la relación que establece el artista con la vida, y por consiguiente, con el espectador. Para ello, comenzaremos haciendo referencia al movimiento futurista, de éste nos interesa destacar para nuestra investigación, sus trabajos en el campo de la performance, alimentada por los ideales de transformación de la vida cotidiana a través del arte, como lo expresaba Marinetti (1876-1944) en uno de sus manifiestos "Gracias a nosotros, llegará un momento en el que la vida ya no 
será una simple cuestión de pan y de trabajo, ni tampoco una vida de ociosidad, sino una obra de arte" (Roldberd, 1996, p. 30). Estos crearon las veladas futuristas y las performance para establecer otro tipo de relación con el espectador, como se describe en el manifiesto de los primeros futurista "Solamente es vital el arte que descubre sus propios elementos en el medio que le rodea" (González, Calvo \& Marchán, 1999, p. 149). Con el trabajo futurista, podemos ver, como el espectador empieza a ser utilizado como un agente activo dentro de la propia práctica artística. El espectador, es reubicado por el artista, como un elemento formal dentro de la composición de la obra. Los artistas futuristas rompieron los límites del arte, se introdujeron en la vida para modificar su percepción, la transformaron en la medida que era utilizada, en el tiempo presente que duraba una velada o una performance pero sin llegar a tener un efecto real de transformación. A pesar de ello, podemos decir que el legado dejado por los futuristas en el mundo del arte, es incuestionable.

De la intención de los artistas futuristas, de romper los límites del arte para transformar la vida cotidiana, pasamos al acto de los cubistas de introducir objetos cotidianos o partes de estos, en sus collages, para investigar las diferencias entre la presentación de la vida y la representación de ésta, este acto que conlleva el concepto de apropiación, proporciona a los artistas, una nueva mirada en su relación con la realidad, una mirada, que se fue ampliando progresivamente durante todo el siglo XX, ante una realidad cada vez más saturada de posibilidades objetuales. Duchamp, es el encargado de desarrollar este acto de apropiación para incluir los conceptos de elección y descontextualización en el mundo del arte. Por su vinculación al dadaísmo, éste utiliza la cotidianidad objetual, para realizar creaciones anti-artísticas pero también tenemos que tener en cuenta sus intenciones, al no querer que la obra provocara ninguna emoción estética, basando su elección con respecto a los readymade, en una ausencia total del gusto. La utilización de materiales considerados como no artísticos y elegidos al azar, y la creación de obras en espacios ajenos al mundo del arte como calles, cafeterías, etc., fue precisamente, lo que llevó a los dadaístas a romper los límites del arte y apropiarse de la realidad.

El ready made derivó en el Objet trouvé de los surrealistas, aunque apoyados sobre un afán más positivo en sus acciones, observamos como el concepto de azar y la tradición provocativa de los dadaístas, vuelve a aparecer. El azar es utilizado como medio para introducirse en la realidad y producir experiencias vivenciales. Con la provocación, el gesto, la elección y el objeto, los surrealistas pretendían cuestionar lo establecido, con la intención de que la gente despertara de su habitual forma de mirar, tanto en el arte como en la vida misma. Con ese propósito, introdujeron también el concepto de "simultaneidad", que jugaría un papel importante en la creación surrealista pues era "entendida como una sucesión sin coordinar, casual, como principio dominante en la vida cotidiana. Aceptándola como principio dominante en la vida y en el arte..." (Marchán, 1997, p.161).

Todos estos nuevos conceptos intención, acto, elección, apropiación, descontextualización, azar, simultaneidad...dieron paso a la segunda mitad del siglo $\mathrm{XX}$, ofreciendo nuevas posibilidades estéticas de aproximación a la vida cotidiana. Unas veces, esas aproximaciones se llevaron a cabo bajo la apropiación crítica de la vida, como en el caso del movimiento Nuevo realismo y otras veces, como pasó con 
el Pop Art, sus aproximaciones al público en general, fueron gracias a las imágenes agradables y fáciles de asumir, que produjeron sobre los objetos consumidos por las masas.

Siguiendo la huella de lo objetual, y por consiguiente, la apropiación de la vida por parte del arte, nos situamos en el assemblage. Utilizando la misma metodología, que anteriormente hemos descrito con los surrealistas al trabajar sus Objet Trouvé, los artistas del assemblage, eligen de forma casual los materiales o fragmentos de objetos diferentes para descontextualizarlos, despojándoles así de su semiótica habitual y poder crear nuevas lecturas. Los artistas cambiaron su lugar habitual de abastecimiento de materiales por tiendas de segunda mano, chatarrerías, basureros, etc.

Tenemos que añadir a esas nuevas lecturas artísticas, otra innovación, la colonización del objeto por el espacio circundante, esto supuso un cambio en la relación habitual del espectador con la obra, de situarse frente a... pasa a ser situado dentro de.... El espectador es situado dentro de los "ambientes" artísticos, considerados como "... la extensión y expansión transitable de la obra en el espacio real. No se trata de una reproducción, sino de la instauración de una realidad en una situación espacial" (Marchán, 1997, p. 173). A este tipo de obras pensadas en función del espacio donde se van a exponer, se les confiere un carácter de experiencia única. La expansión de la obra en el espacio, ampliaba la apropiación del arte sobre la vida.

Del interés ambiental, donde los objetos son descontextualizados y colonizan un espacio real en el que el espectador puede penetrar dentro de él, pasamos al happening, donde el espectador se convierte en agente activo dentro de la propuesta artística, pues la interacción del espectador con la obra es fundamental para la creación y desarrollo de ésta, ya que la esencia del happening, es que sea una vivencia, por esta razón, destacamos su vinculación con la realidad. Según Marchán (1997), una de las primeras oportunidades para que un público más amplio asistiera a actos vivos, la proporcionó Allan Kaprow, con la realización de su obra 18 happening en 6 partes, realizado en Nueva York en 1959 y expuesto en la Reuben Gallery.

De Kaprow, pasamos a uno de los referentes más directos para nuestro trabajo, Joseph Beuys. Este consideraba que las acciones ocupan un lugar prioritario, pues pensaba que "Todas las Acciones, eran importantes para ampliar el viejo concepto del arte. Para ampliarlo y engrandecerlo cuando fuera posible, de forma que pudiera abarcar cualquier actividad humana" (Arici et al., 1984, p. 27). Para esta investigación nos interesan sus ideales sociales vinculados a la educación y su metodología, pues sus clases se alejaban del tipo de práctica académica tradicional y se encontraban más próximas a las acciones y la performance. En sus exposiciones orales durante las clases, llenaba las pizarras con signos, iconos y diagramas que han llegado a considerarse como obras de arte autónomas. Queremos destacar una diferencia que consideramos fundamental, entre su trabajo artístico-docente y nuestra investigación, el contexto académico donde Beuys planteaba sus trabajos, estaba vinculado al mundo del arte pues era una Academia de Bellas Artes, en cambio, el nuestro, se ha desarrollado también en un contexto académico pero ajeno al arte. Esta diferencia la consideramos fundamental para crear una aproximación más eficaz, entre el arte y la vida cotidiana. 
Las diferentes aproximaciones a la vida que los artistas han estado realizando a lo largo del siglo XX, nos sitúan, en el concepto de desmaterialización del objeto de arte (Parcerisas, 2007, p.15). En la década de los sesenta y los setenta, las propuestas artísticas que se desarrollaban bajo ese concepto de desmaterialización, exigían materiales efímeros como el tiempo, el lenguaje, actos, etc. A esa concepción de lo efímero, tenemos que añadir la concepción de la realidad presente, en la que se reúnen al artista, la obra y el público en un mismo momento, en el momento del proceso creativo. Este tipo de acciones artísticas happening, Fluxus, festivales, etc. que en su momento se denominaban de forma general eventos, pasaron posteriormente a utilizar el término de performance (Torrens, 2007, p. 11), como denominación genérica. También el término Body Art o arte del cuerpo, fue considerado otra opción dentro de la acción. En este tipo de arte, vemos como el cuerpo se convierte en un sistema de signos. El papel del artista pasa a ser de coordinación, convirtiéndose en catalizador de la acción creativa. La acción exige, una ampliación de la sensibilidad artística por parte del espectador, como nos expone Aznar Almazán en su libro El arte de acción, "la actitud del espectador tiene que ser de observación participante, en un oscilar continuo entre el dentro y el fuera de los sucesos: por un lado, debe atrapar empáticamente el sentido de unos acontecimientos y unos gestos específicos; por el otro, debe dar un paso atrás para situar esos significados en contextos más amplios y que así adquiera un sentido más profundo o general" (Aznar, 2000, p. 8). La performance al ser poliforma y polisémica, estimula el pensamiento creativo, vinculando al performen y al público, en la creación y percepción de un presente.

En el espacio relacional que se genera cuando se realiza una performanece, la comunicación kinestésica percibe el propio cuerpo y el cuerpo del otro, articulada en el tiempo y en el espacio. La performance al ser poliforma y polisémica crea una comunicación de creación y percepción instaurada en un presente. También amplia los límites del significado y del significante, creando un espacio a la indeterminación. La performance y la vida están vinculadas, pues se basan en los mismos elementos que las estructuran, la presencia, el espacio y el tiempo, concretados en un ciclo vital de principio y fin. Esta afinidad con la vida, se convierte en presentación de la acción performativa y es lo que la diferencia del concepto representación.

Con los referentes anteriormente expuestos, hemos ido comprobando que el propósito de unión entre el arte y la vida que el artista ha ido realizando, a través de su intención, acto y expansión por el espacio circundante, ha sido de apropiación de la vida. Es evidente, que con este tipo de propuestas, se abren otros espacios alternativos y más vinculados con la realidad cotidiana, estableciéndose otras formas de relación entre la obra y el público pero la duda que nos surge es, hasta qué punto, el público llega a interpretar la expresión del artista durante una performance para que se establezca una interacción comunicativa, y por consiguiente, una aproximación real con la vida cotidiana.

\section{El arte como expresión social y su necesaria interpretación}

Para ayudarnos a despejar la duda sobre si realmente se establece una interacción comunicativa entre el público y el arte de acción, Martín Algarra en su libro Teoría de la comunicación: una propuesta (Martín, 2008), nos indica que para llegar 
a una interacción comunicativa se tienen que realizar las dos acciones básicas: la expresión comunicativa y la interpretación de esa expresión. Este autor considera la comunicación como una relación en la que se comparten contenidos cognitivos, con el fin de significar. Reflexionando sobre la naturaleza transaccional de la comunicación, ésta necesita algo que compartir, la intención de compartir, alguien con quien compartir y las acciones de los que comparten: la expresión y la interpretación. Durante la comunicación hay siempre tres tipos de elementos: subjetivos, objetivos y práxicos. En los elementos práxicos, la expresión es el primer elemento de la comunicación. Es normal que pensemos que todo lo que hay en el mundo expresa algo pero Martín (2008) nos ayuda a distinguir la expresión del significado, utilizando dos conceptos: expresividad y expresión, definiendo su diferencia de la siguiente manera:

La expresividad es ese carácter epifánico de todos los objetos del mundo (incluido el hombre los productos de sus acciones)... La expresividad no es algo distinto del propio objeto del mundo: es el objeto en cuanto que perceptible e interpretable por otros.

La expresión se da a través de los objetos del mundo, pero no es una cualidad de ellos. Se basa en el carácter epifánico de los objetos y de su apariencia identificadora... La expresión es una acción, lo cual implica un autor que se expresa. La expresión es una acción significativa que tiene como finalidad manifestar lo que se piensa o se siente. Por tanto, la finalidad de la expresión es precisamente el significado. (Martín, 2008, p. p.143144).

Martín nos ha especificado la diferencia entre expresividad y expresión, concretando el concepto de expresión como la acción que consiste en plasmar un significado. Este autor nos sigue afinando la expresión comunicativa y la vuelve a dividir en dos tipos: la expresión solitaria y la expresión social:

La expresión solitaria es la mera manifestación de un conocimiento o un sentimiento. En ella no se busca nada que no sea la plasmación de un significado. El fin de esa acción es el propio producto expresivo. La expresión social es también la manifestación de un conocimiento o un sentimiento. Pero la finalidad de su expresión es que otros lo conozcan. El producto es un instrumento para alcanzar el verdadero fin de la expresión social: la comprensión, la comunicación. (Martín, 2008, p. 145).

Ahora pasamos a la segunda acción de la interacción comunicativa, la interpretación. Martín divide también este concepto, entre la interpretación de la expresividad y la interpretación de la expresión. Sin entrar en una descripción extensa sobre la interpretación de la expresividad, ésta consiste básicamente en percibir e identificar las cosas que nos rodean, ya sean objetos naturales u objetos artificiales, para después comprender su naturaleza. La expresión, como antes hemos expuesto, es dividida por Martín, en solitaria y social, por esa razón, su interpretación también es independiente. En la interpretación de la expresión solitaria es el producto significativo el que hay que interpretar. Pero como el producto expresivo solitario se creó, con la indiferencia de ser o no ser interpretado pues significa lo que es, aunque se le interpretara, no añadiría nada a la acción y al producto. En cambio, en la interpretación de la expresión social, es fundamental que el significado llegue a otro, 
a través, del producto. También tenemos que tener en cuenta, que el producto tiene que ser interpretado según su finalidad social pues ha sido creado bajo la condición de eficacia que se pretende para su finalidad.

Considerando el arte como expresión social y basándonos en la necesidad de su interpretación para que cumpla su finalidad, es por lo que en la parte práctica de nuestra investigación y que expondremos en el siguiente apartado, desarrollamos el proceso de un caso práctico de aproximación entre el arte de acción y la vida cotidiana de los estudiantes de Educación Infantil para comprobar las dificultades que existen para llegar a interpretar el arte de acción y si se llega a producir una interacción comunicativa real entre ambas partes.

\section{Desarrollo de un caso práctico para llevar a cabo posibles aproximaciones entre el arte de acción y la Educación Infantil en la vida cotidiana educativa}

En este apartado se desarrollarán las dos fases establecidas para este proyecto de investigación. Las dos primeras fases, se llevaron a cabo en seis sesiones experimentales seguidas y con un intervalo temporal

\subsection{Contextualización, características del alumnado/espectador y aparatos utilizados para registrar las experiencias}

Nuestro trabajo está contextualizado dentro del campus de la Universidad de Alicante. Uno de los espacios utilizados para la investigación es la Facultad de Educación, departamento de Didáctica general y Didáctica específica, área de Expresión Plástica.

Las características del aula de plástica donde hemos trabajado son las siguientes: de forma rectangular y diáfana, consta de una zona reservada para el docente y resaltada por una tarima de madera. Las restantes tres cuartas partes del aula, están destinadas para los alumnos y se caracteriza por la disposición lineal de las mesas de dibujo y las sillas. En los laterales y en el fondo del aula podemos encontrar varios armarios, cuatro mesas rectangulares y largas, dos fregaderos y grandes ventanales que proporcionan abundante luz natural.

Para una de las fases experimentales planteadas, nos hemos servido del espacio circundante al aula, es decir, el Campus universitario. Las características de éste son: amplias zonas ajardinadas que proporcionan descanso a los-as alumnos-as, al profesorado y los visitantes, edificios con diferentes arquitecturas, entre ellas destacamos el museo de la universidad MUA, por su edificación a ras del suelo.

Este trabajo se ha realizado con un total de 110 alumnas/os de primer curso de Educación Infantil, del título de Maestro, en el curso académico 2009-2010, matriculados en la asignatura de Dramatización y títeres. La edad de los alumnas/os estaba comprendida entre los 19 y 25 años. Durante el proceso de investigación el número de alumnas/os durante las clases varió según los días, pero siempre oscilaron entre un grupo de 110 alumnas/os, que a su vez se subdividía en otros dos, compuesto por 50 o 60 alumnos. Para poder llevar a cabo las propuestas artísticas, la totalidad del alumnado se subdividió en grupos con un mínimo de 5 alumnas/os o un máximo de 9 alumnas/os. También, en este caso, la variable del sexo es un dato a tener en 
cuenta, ya que el grupo estaba compuesto por mujeres, a excepción de dos de sus miembros que eran hombres.

Para registrar todos los procesos planteados en este trabajo de investigación, se utilizaron simultáneamente, una cámara de vídeo marca Sony Handycam Mini DV mod. DCR-HC27E PAL y una cámara de fotografiar digital marca Canon EOS20D. Estos medios nos han permitido tener una amplia documentación sobre las conductas realizadas.

\subsection{Primera fase del proceso}

Esta primera fase fue diseñada para concretar si existían conocimientos previos dentro de un contexto cotidiano sobre la percepción de la performance como acción vinculada al arte.

Para llevar a cabo la medición de la variable dependiente conocimientos previos, mediante la manipulación de las variables independientes, se emplearon dos sesiones y media, en las que se trabajó de la siguiente manera:

1. Realización de la primera performance para romper el hábito conductual entre los roles estereotipados dentro del contexto académico universitario, donde estábamos trabajando y así establecer otro tipo de relación.

2. Se pasó un pre-test a al alumnado/espectador para saber cuál era su nivel de conocimiento sobre el arte contemporáneo y más concretamente, sobre el arte de acción.

3. Manipulación de los estímulos ambientales, a partir de crear una propuesta de acción distinta para cada día que tuviéramos que coincidir con el alumnado/ espectador en el espacio predeterminado. En este trabajo, solamente vamos a mostrar tres de estas acciones, como referencia, de las acciones que se han estado llevando a cabo para la investigación.

4. Propuesta de arte de acción para crear una interacción con los/as alumnos-as/ espectadores, convirtiéndose así en parte activa de la acción.

\subsubsection{Performance I}

Nuestra primera acción (Fig. 1), se pudo llevar a cabo por el desconocimiento de nuestra identidad por parte del alumnado/espectador, pues pertenecían al primer curso de Magisterio Infantil, el único vínculo que teníamos previamente establecido era el día y el espacio. Antes de la hora prevista para el comienzo de la presentación de la asignatura, nos introdujimos en el espacio vacío y colocamos la cámara de vídeo en un lugar determinado para que pudiera coger una buena panorámica pero teniendo en cuenta, que no estuviese muy visible. Nos situamos dentro del espacio destinado para el alumnado/espectador, para crear otro tipo de relación antes de revelar nuestra identidad, y que por el hábito social, se llevaran a cabo los roles preestablecidos entre docente y alumnado. $\mathrm{Al}$ ser un espacio con unas dimensiones bastante amplias, escogimos situarnos en una zona intermedia, ni muy cerca de la zona reservada para el profesor, ni muy alejada, pues llamaría demasiado la atención. La actitud adoptada fue de espera, utilizando como elementos de apoyo una carpeta sobre la mesa y un cuaderno, que nos permitían, a su vez, ir anotando las percepciones que estábamos 
viendo y sintiendo. Esta actitud nos ayudó a establecer una fusión con el entorno y poder hacer un registro conductual, observando que los-as alumnos-as/espectadoresas, se iban introduciendo en el espacio vacío, con ritmos aleatorios. Al ser su primer encuentro con dicho espacio, la duda por la decisión de su ubicación, generaba la necesidad de establecer una relación visual con el entorno, para elegir su asiento según sus preferencias. Observamos que los que menos dudaban, eran los-as que tenían claro que les gusta estar en primera fila o al final.

Tras observar ese comportamiento conductual y su relación con el espacio, comenzamos a establecer contacto con las que se habían situado a nuestro alrededor, este contacto podía ser verbal o visual, al mismo tiempo, prestábamos especial interés a los diálogos que iban surgiendo sobre la posible identidad del docente, y sobre todo, en sus comentarios hacia el retraso que estaba habiendo en la presentación de la asignatura. Dentro de ese ambiente generado por la impaciencia y el desconcierto, comenzamos a recoger los elementos que habíamos utilizado sobre la mesa (carpeta, cuaderno, bolígrafos....) y nuestros objetos personales. Durante ese proceso, nuestro umbral de percepción auditiva se agudizó para percibir el sonido ambiental ocasionado por las voces, éste algunas veces creaba una capa homogénea en el espacio, por el grado de intensidad que alcanzaba, fue por ese motivo, que decidimos añadir a ese intenso campo gravitatorio sonoro generado por la impaciencia, el sonido producido por el impacto de nuestro calzado sobre el suelo, cuando nos desplazamos por uno de los laterales del espacio para dirigirnos hacia el lugar establecido para el docente. Esa acción, consiguió alterar el umbral de percepción del alumnado/espectador, ya que percibimos que la vibración casi homogénea predominante producida por el sonido ambiental de voces y movimientos, fue disminuyendo progresivamente hasta que cesó casi por completo al subirnos a la tarima, dejar nuestros elementos sobre la mesa y la silla correspondiente y situarnos frente a ellos-as. Esa homogeneidad del sonido se transformó por la emoción de la sorpresa, en una serie de secuencias sonoras fragmentadas y dispersas por la estancia. Durante un periodo de tiempo determinado, el tipo de relación creada entre el docente-artista y el alumnado/espectador fue distinta por nuestra acción y ubicación en el aula, nos situamos dentro de y no frente $a$. El recorrido de dentro hacía fuera, nos permitió establecer desde el inicio otra forma de relación.
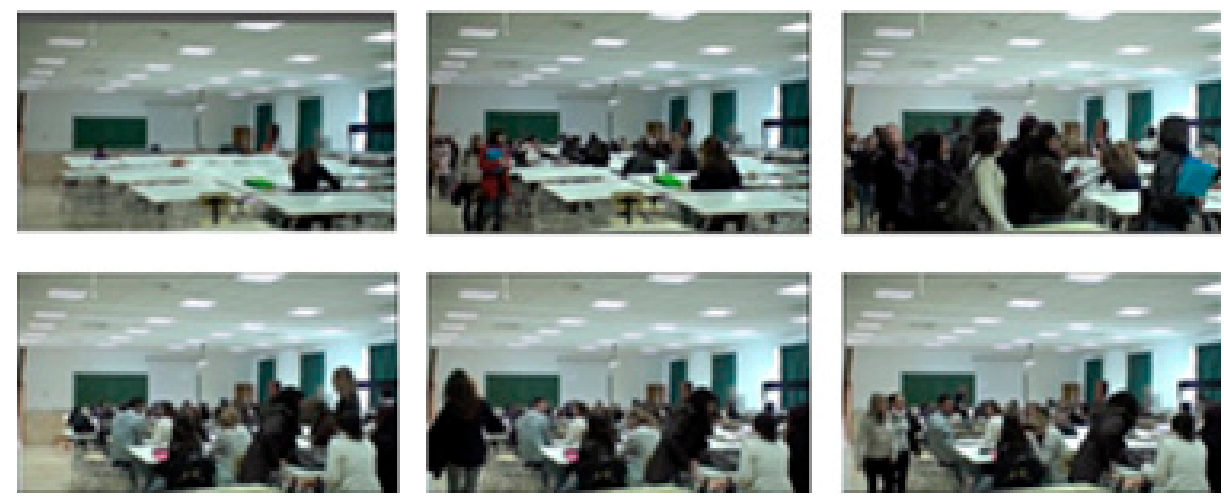

Figura 1. Performance I. Facultad de Educación de la Universidad de Alicante, 2010. (Clips de vídeo. Fuente: propia). 


\subsubsection{Pre-test realizado en el contexto cotidiano}

Una de las características de los diseños cuasiexperimentales, es que no haya selección aleatoria de los participantes, ello puede ocasionar, que alguna variable extraña pueda influir en los resultados finales, si bien, para controlar esta posible influencia, se pasó un pre-test inmediatamente después de realizar la primera performance:

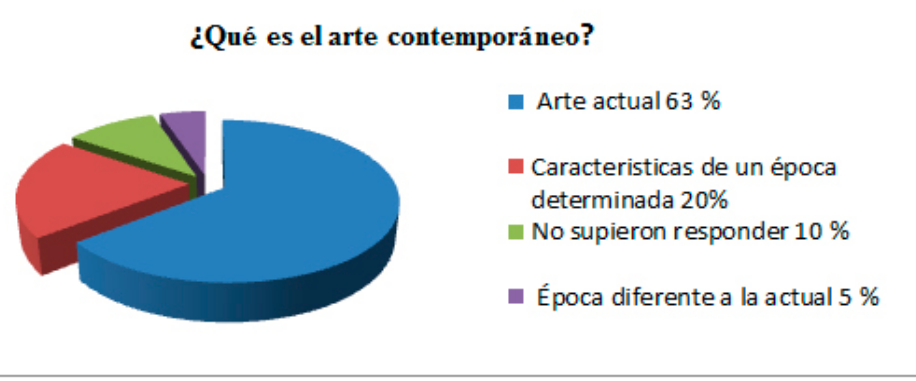

Figura 2. Gráfico en el que se muestra el resultado de la primera pregunta del pre-test para comprobar el nivel de conocimientos del alumnado/espectador sobre el arte contemporáneo.

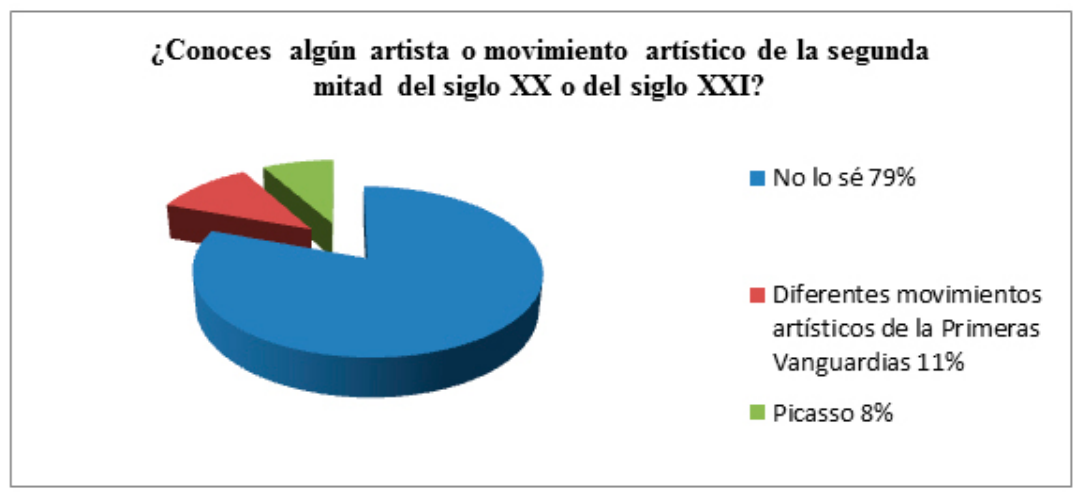

Figura 3. . Gráfico en el que se muestra el resultado de la segunda pregunta del pre-test para comprobar el nivel de conocimientos del alumnado/espectador sobre el arte del siglo XX y XXI.

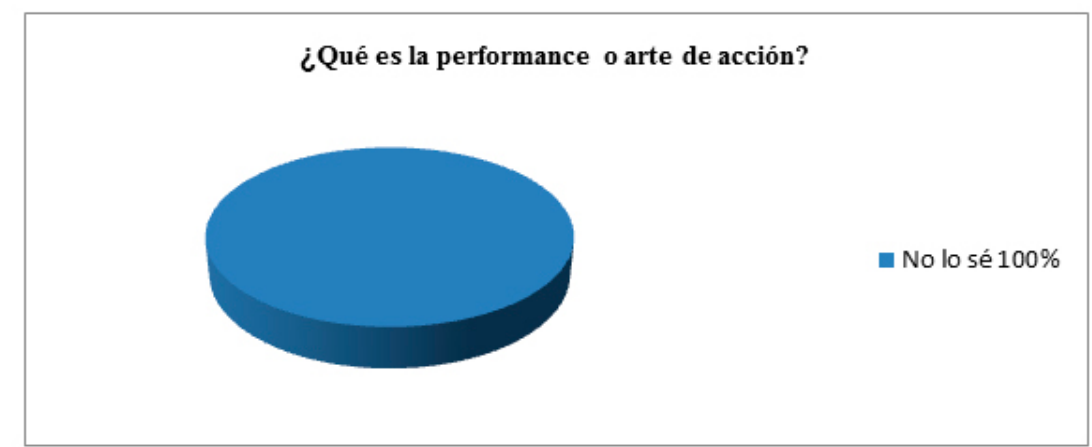

Figura 4. Gráfico en el que se muestra el resultado de la tercera pregunta del pre-test para comprobar el nivel de conocimientos del alumnado/espectador sobre la performance o el arte de acción. 
A través de este pre-test, se comprobó el nivel de conocimientos del alumnado/ espectador sobre el arte del siglo XX y XXI, y especialmente, sobre el arte de acción, que dio en esta última pregunta un resultado homogéneo de desconocimiento.

\subsubsection{Performance II}

Tras conocer los resultados del pre-test, y comprobar que el desconocimiento sobre el arte de acción, era homogéneo en todo el alumnado/espectador, en el siguiente encuentro realizamos la segunda performance. Nuestra acción fue recibirles con una máscara hecha con espejos (Fig. 5). Sus rostros, su identidad eran reflejados en la zona de nuestra identidad. La observación directa y los registros audiovisuales de sus caras y gestos, nos dejaba claro que se estaba estableciendo un contacto, basado en la sorpresa y en lo anecdótico, pues el concepto de acción relacionado con el arte, no estaba aún establecido.
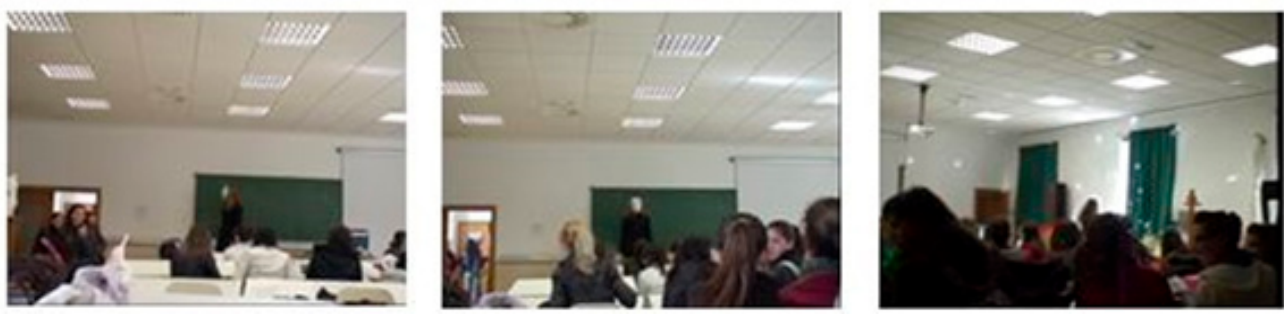

Figura 5. Performance III. Facultad de Educación de la Universidad de Alicante, 2010.

(Clips de vídeo. Fuente: propia).

\subsubsection{Performance III}

En esta tercera performance, les recibimos con una máscara de escayola, pero en esta ocasión, les ofrecimos unos bolígrafos para que se expresaran y poder interaccionar sobre la zona de la identidad, el alumnado/espectador se estaba convirtiendo en parte activa de la acción (Fig.6). A través de este planteamiento más interactivo, los registros de observación y tecnológicos nos aportaron datos más concretos sobre la variable dependiente conocimiento previo.

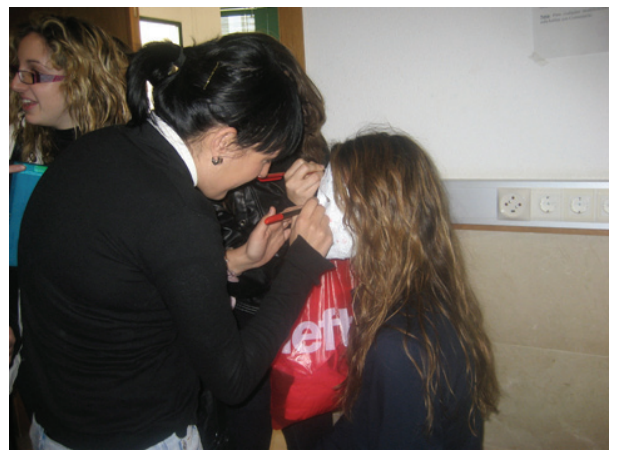

Figura 6. Performance III. Facultad de Educación de la Universidad de Alicante, 2010. (Clips de vídeo. Fuente: propia).. 
Queremos añadir, que éramos conscientes del riesgo de la relación evidente entre la máscara y el teatro y por consiguiente, su familiaridad con el concepto de representación pero precisamente eso es uno de los retos que nos planteamos, utilizar un objeto con esa carga y poder separarla del teatro para conseguir presentarla y no representarla.

\subsubsection{Conclusiones de la primera fase del proceso}

\begin{tabular}{|l|l|l|l|}
\hline \multicolumn{4}{|c|}{ Medición de la variable dependiente conocimientos previos } \\
\hline $\begin{array}{l}\text { Manipulación de las } \\
\text { variables independientes }\end{array}$ & \multicolumn{1}{|c|}{ Performance I } & \multicolumn{1}{|c|}{ Performance II } & Performance III \\
\hline $\begin{array}{l}\text { Resultados de la } \\
\text { observación conductual } \\
\text { directa y posterior } \\
\text { visionado del material } \\
\text { audiovisual }\end{array}$ & $\begin{array}{l}\text { Los registros } \\
\text { observacionales de sus caras } \\
\text { y gestos, nos dejaba claro } \\
\text { que se estaba estableciendo } \\
\text { un contacto, basado en la } \\
\text { sorpresa y en lo anecdótico. }\end{array}$ & $\begin{array}{l}\text { Seguimos observando } \\
\text { que el concepto de } \\
\text { presentación de la } \\
\text { acción relacionado con } \\
\text { el arte, no estaba aún } \\
\text { establecido. }\end{array}$ & $\begin{array}{l}\text { El alumnado/espectador } \\
\text { comenzó a entrar en el } \\
\text { espacio, con } \\
\text { incertidumbre y } \\
\text { curiosidad pero sin tener } \\
\text { datos cognitivos previos, } \\
\text { observamos quela } \\
\text { performance ejecutada } \\
\text { en un contexto cotidiano } \\
\text { no es percibida, ni } \\
\text { interpretada como una } \\
\text { acción vinculada al arte. }\end{array}$ \\
\hline
\end{tabular}

Figura 7. Tabla en el que se muestran los resultados de la primera fase del proceso.

El registro de la observación conductual directa y los medios tecnológicos que utilizamos para registrar la realización de las tres performances que hemos expuesto, nos corroboraron el resultado del pre-test pero también nos demostró que la performance ejecutada en un contexto cotidiano y sin un conocimiento previo, el público no percibe, ni interpreta la performance como acción vinculada al arte.

\subsection{Segunda fase del proceso}

En la primera fase de esta investigación, hemos comprobado que sin el conocimiento previo necesario y ejecutada en un contexto cotidiano, el alumnado/ espectador no percibe, ni interpreta la performance como acción vinculada al arte, por lo tanto, la segunda fase de nuestra trabajo, fue diseñada para los-as mismos-as alumnos-as/espectadores-as que nos habían demostrado que no tenían conocimientos previos sobre la performance. Desde ese desconocimiento, en esta segunda fase, se le propone a dichos sujetos experimentales, la adquisición de datos cognitivos y la vivencia de la experiencia, mediante el arte de acción, para saber qué dificultades tienen para llegar a entender la diferencia entre los conceptos presentación (acción) y representación, concretando así, en qué fase del proceso planteado es integrada dicha diferencia.

Al igual que en la primera fase para llevar a cabo la medición de las variables dependientes datos cognitivos y vivencia de la experiencia por medio de la manipulación de las variables independientes, se emplearon en esta ocasión tres sesiones y media, en las que nuestro trabajó se desarrolló de la siguiente manera: 
1. Exposición de la información para aportar los datos cognitivos necesarios sobre arte contemporáneo, y más concretamente, sobre el arte de acción.

2. Propuestas de actividades perceptivas para sensibilizar al público sobre su cuerpo y el de los demás.

3. Propuesta de arte de acción para que los/as alumnos-as/espectadores-as tengan la vivencia de la experiencia artística. Práctica sobre el concepto "presentación".

4. Creación y realización de improvisaciones sobre entornos cotidianos. Práctica sobre el concepto de "representación".

\subsubsection{Proceso de desarrollo performativo para los/as alumnos-as/espectadores-as. Diferencia entre presentación y representación}

El desarrollo práctico planteado para esta segunda fase de nuestro trabajo de investigación, va paralelo a la exposición teórica para aportar los datos cognitivos necesarios sobre los cambios realizados en el arte del siglo XX, y más concretamente, sobre el arte de acción. Aunque es simultánea la manipulación de las variables independientes, en la medición de la variable dependiente datos cognitivos, se puede concretar su resultado independientemente de la medición de la variables dependiente vivencia de la experiencia, por lo tanto, a pesar de la aportación de los datos cognitivos sobre el arte de acción, el alumnado/espectador seguían sin llegar a comprender el concepto de acción dentro del arte, aunque tenemos que añadir, que la información aportada generó nuevas redes neuronales que permitieron una vinculación con el arte contemporáneo, que antes no existía.

Habiendo concretado el resultado de la variable dependiente datos cognitivos, el cual nos evidencia que el concepto de acción/presentación aún no se entiende, el proceso de desarrollo performativo que paralelamente habíamos planteado para los/as alumnos-as/espectadores-as basándonos en una de las performance que habíamos realizado y expuesto anteriormente, sigue siendo impuesto. Bajo nuestra guía, comenzamos la creación de un objeto común, en este caso, una máscara de escayola (Fig.3.B) Como estábamos trabajando la percepción, antes de comenzar su elaboración por parejas, les pedimos que se tocaran su propia cara con los ojos cerrados (Fig.3.A), y después, la de su compañera/ro para empezar a percibirse y a relacionarse en ese contexto, de otra manera.
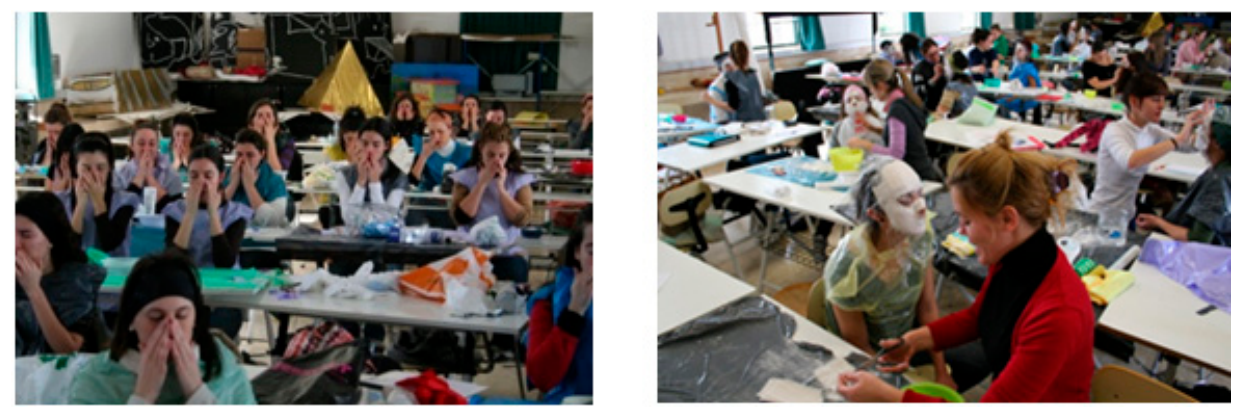

Figura 8. (a) Proceso perceptivo y (b) técnico, realizado por los/as alumnos-as de Magisterio de Educación Infantil. Facultad de Educación de la Universidad de Alicante, 2010.

(Fotografía. Fuente: propia). 
Tras la realización de la máscara y dentro de este proceso creativo de percepción, se llevó a cabo la siguiente propuesta de interacción. Recogimos y colocamos todas las máscaras de escayola en el suelo, dispuestas en círculo, sin que los/as alumnosas/espectadores-as supieran en qué lugar del círculo se había colocado su máscara, después se les pidió que se desplazaran en fila alrededor del círculo de máscaras hasta identificar su faz (Fig. 4), en el momento que creyeran que la habían identificado, se tenían que introducir en el interior del círculo frente al objeto. El proceso siguió hasta que todas estuvieron en el interior del círculo pero en este caso un grupo de diez personas no consiguieron identificarse, por esa razón, se les pidió a las que estaban seguras que comprobaran si habían acertado en su identificación, solamente cinco fallaron, de un grupo formado por 40 miembros. Uniendo a las no se identificaron y a las que lo hicieron erróneamente, se formó un grupo de quince personas, con este grupo, se volvió a repetir el proceso, al final cinco personas no consiguieron identificar su faz. Con este trabajo se esforzaron por percibir su imagen o lo que creían conocer de sus rasgos. También la relación con el espacio y con los demás cambió pues ya no estaban detrás de una mesa sino que se movían por el espacio e interaccionaban con los demás con un propósito distinto al habitual.

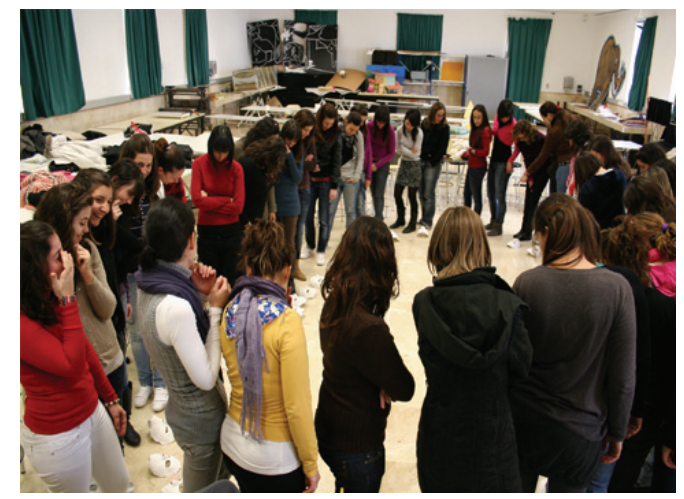

Figura 9. Proceso de identificación, realizado por los/as alumnos-as de Magisterio de Educación Infantil. Facultad de Educación de la Universidad de Alicante, 2010. (Fotografías. Fuente: propia).

\subsubsection{Práctica sobre el concepto presentación}

A partir de las prácticas de percepción, creación y reconocimiento del objeto creado, la siguiente propuesta se basó, en la repetición por parte del alumnado/ espectador de la tercera performance que habíamos realizado en el aula. Consistía en salir al campus universitario y que pidieran al público su intervención en la zona de la identidad, escribiendo o dibujando sobre la máscara, con esa interacción se generó otro público (Fig.5 A-B). Aunque siguió siendo un proceso creativo establecido por nosotros, pues los/as alumnos-as/espectadores-as aún no se habían convertido en productores, es decir, llegar a la forma formada (Eco, 2002) pues habían tenido un proceso de expresión fundamentalmente técnica y no habían llegado a tener una experiencia estética completa, esta propuesta nos sirvió para comprobar si a través de la vivencia de la experiencia artística se establecía mejor la diferencia entre presentación (acción) y representación. 


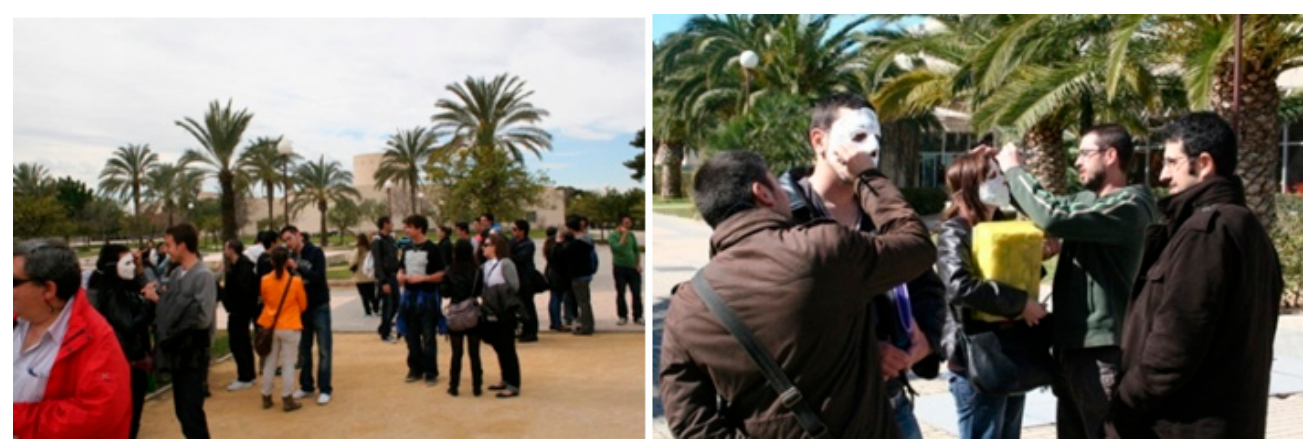

Figura 10. (a y b) Propuesta de arte de acción, realizada por los/as alumnos-as de Magisterio de Educación Infantil. Campus de la Universidad de Alicante, 2010. (Fotografías. Fuente: propia).

\subsubsection{Práctica sobre el concepto de representación}

Después de manipular las variables independientes, a través, de llevar a cabo la vivencia de la propuesta de arte de acción, comprobamos con preguntas directas si se había llegado a percibir, a través de la experiencia, la diferencia entre presentación (acción) y representación, aunque por las respuestas se percibía un intento de comprensión, era evidente que la diferencia aún no estaba clara. El resultado de estos procesos nos supuso el planteamiento de un ejercicio que no teníamos introducido dentro de este proceso pero que nos daría la posibilidad de contrastar resultados, dicha propuesta consistía en realizar una improvisación que representara un contexto cotidiano, es decir, una puesta en escena. El alumnado/espectador no tuvo ningún tipo de dificultad a la hora de improvisarla, crearla y ejecutarla, les resulto fácil y divertido representar los estereotipos que vemos habitualmente por los medios de comunicación. Precisamente esa fluidez, nos hizo reflexionar sobre el tema del hiperdesarrollo del lenguaje visual que existe en la actualidad y que predispone a ubicar todo tipo de acción, dentro de una concepción de representación.

\subsubsection{Conclusiones de la segunda fase del proceso}

Como ya hemos expuesto en el desarrollo de esta segunda fase, con la medición de la variable dependiente datos cognitivos pudimos observar que a través solamente de la aportación de los datos cognitivos, el alumnado/espectador no llegaba a entender la diferencia entre presentación (acción) y representación.

Las propuestas intermedias del proceso perceptivo, técnico y de identificación, nos ayudaron a desarrollar una cierta sensibilización en el alumnado/espectador, pero fueron las prácticas sobre los conceptos de presentación (acción) y representación las que nos aportaron un resultado más concreto. La medición de la variable dependiente vivencia de la experiencia, es decir, la experiencia artística mediante el arte de acción, dejó la semilla de la vivencia pero el alumnado/espectador seguía sin llegar a entender el concepto de presentación (acción). La siguiente práctica sobre el concepto de representación fue decisiva, inmediatamente después de haber realizado la improvisación, y habiendo observado la fluidez en su creación y ejecución, le 
planteamos la siguiente pregunta: ¿a quién estabais representando cuando se planteó la experiencia artística de la performance en el campus universitario? la contestación fue unánime, a nadie. La reflexión sobre esta respuesta favoreció que se llegara a entender la diferencia entre los conceptos de presentación (acción) y representación.

\begin{tabular}{|c|c|c|c|c|}
\hline \multicolumn{5}{|c|}{$\begin{array}{c}\text { Segunda fase del proceso } \\
\text { Medición de las variables dependientes datos cognitivos y vivencia de la experiencia }\end{array}$} \\
\hline $\begin{array}{l}\text { Manipulación } \\
\text { de las variables } \\
\text { independientes }\end{array}$ & $\begin{array}{l}\text { Aportación delos } \\
\text { datos cognitivos } \\
\text { necesarios sobre el } \\
\text { arte de acción }\end{array}$ & $\begin{array}{l}\text { Propuestas de } \\
\text { actividades } \\
\text { perceptivas }\end{array}$ & $\begin{array}{l}\text { Práctica sobre el } \\
\text { concepto } \\
\text { "presentación" }\end{array}$ & $\begin{array}{l}\text { Práctica sobre el } \\
\text { concepto de } \\
\text { "representación" }\end{array}$ \\
\hline $\begin{array}{l}\text { Resultados de la } \\
\text { observación } \\
\text { conductual } \\
\text { directa y } \\
\text { posterior } \\
\text { visionado del } \\
\text { material } \\
\text { audiovisual }\end{array}$ & $\begin{array}{l}\text { Con la aportación de } \\
\text { los datos cognitivos } \\
\text { solamente, el } \\
\text { alumnado/espectador } \\
\text { no llega a entender la } \\
\text { diferencia entre los } \\
\text { conceptos de } \\
\text { presentación (acción) } \\
\text { y representación. }\end{array}$ & $\begin{array}{l}\text { Se aumenta la } \\
\text { sensibilización del } \\
\text { alumnado/espectador } \\
\text { sobre su cuerpo y el de } \\
\text { los demás }\end{array}$ & $\begin{array}{l}\text { Los sujetos } \\
\text { experimentales } \\
\text { siguen sin llegar } \\
\text { a entender } \\
\text { totalmente la } \\
\text { diferencia entre } \\
\text { el concepto de } \\
\text { presentación } \\
\text { (acción) y } \\
\text { representación. }\end{array}$ & $\begin{array}{l}\text { La reflexión y contraste } \\
\text { dela información } \\
\text { adquirida en las } \\
\text { prácticas sobre los } \\
\text { conceptos de } \\
\text { presentación (acción) y } \\
\text { representación les } \\
\text { permite entender la } \\
\text { diferencia entre ambos } \\
\text { conceptos }\end{array}$ \\
\hline
\end{tabular}

Figura 11. Tabla en el que se muestran los resultados de la segunda fase del proceso.

\section{Conclusiones generales}

El diseño cuasiexperimental que enmarca nuestro trabajo de investigación, nos permite decir que estamos ante conclusiones no concluyentes pero que sí aportan unos datos a tener en cuenta para establecer aproximaciones entre el arte de acción y la vida cotidiana educativa.

Con todo ello, consideramos que la performance ejecutada dentro de un contexto cotidiano, el público en general de manera intuitiva no puede percibir e interpretar la performance como acción vinculada al arte, y por consiguiente, tampoco pueden diferenciar los conceptos de presentación (acción) y representación. Considerando el arte como una forma de expresión social, creemos que para conseguir una aproximación real entre el arte de acción y la vida cotidiana, y así, completar el proceso de interacción comunicativa, primero, tendríamos que solucionar la falta de contenidos cognitivos que tiene el público en general sobre el arte de acción y facilitar su posterior vivencia, para que esta expresión social pudiera llegar a significar y ser interpretada conscientemente, en lugar de ser rechazada o ignorada. Creemos que dicha labor adquiere una gran relevancia cuando nos adentramos en el contexto educativo, ya que en éste, no solo posibilita nuevos procesos cognitivos sino que sería la plataforma principal de proyección a la sociedad y cultura actual, generándose también la posibilidad de transformar "la pedagogía como acto de representación a la pedagogía como acto performativo" (Ellsworth, 2005, p. 128). 


\section{Referencias}

Araci, L., Bezzola, T., Bohelen, M., Christoph, B., Graevenitz, A., Kramer, M., Kurzmeyer, R., Lerm C., Leutgeb, D., Livereiro, C., Loers, V., Martín, A., Pohl, K., Schneede U., Shulz, H., Steiner, J., Stüttgen, J., Szeemann, H., Thönges, R., Voigt, K. (1994): Joseph Beuys. Beuysnobiscum, conceptos desde Academia a Warhol. Madrid: Centro de Arte Reina Sofía.

Aznar, S. (2000): El arte de acción, Arte hoy. Madrid: Nerea.

Efland, D. (2002): Una historia de la educación del arte. Barcelona: Paidós.

Ellsworth, E. (2005): Posiciones en la enseñanza. Madrid: Akal.

García, C. (2001): Investigación cuasiexperimental I: diseños preexperimentales $y$ diseños cuasiexperimentales con grupo de control no equivalente. A: Diseños de investigación en psicología. Madrid: Universidad Nacional de Educación a distancia.

González, Á., Calvo F., Marchán S. (1999): Escritos de arte de vanguardia 1900/1945. Madrid: Ediciones Istmo S.A.

Martín, M. (1997): Teoría de la comunicación: una propuesta. Madrid: Editorial Tecnos.

Marchán, S. (2008): Del arte objetual al arte de concepto. Madrid: Ed. Akal.

Parcerisas, P. (2007): Conceptualismo(s) poéticos, políticos y periféricos. En torno al arte conceptual en España, 1964-1980. Madrid: Akal/Arte contemporáneo.

Roldberd, R. (1996): Performance Art. Barcelona: Ed. Destino.

Torrens, V. (2007): Pedagogía de la performance. Programa de cursos y talleres. Diputación Provincial de Huesca. Edición de Valentín Torrens. 